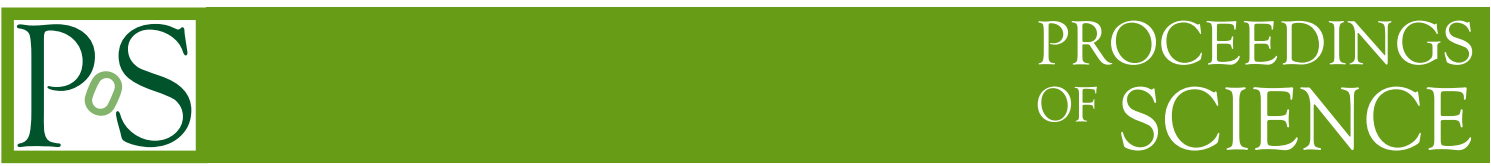

\title{
Status of the short-baseline near detector at Fermilab
}

\author{
Varuna Meddage ${ }^{a, *}$ on behalf of the SBND collaboration \\ ${ }^{a}$ Department of Physics, University of Florida, \\ 2001 Museum Rd, Gainesville, FL 32611, USA \\ E-mail: vmeddage@ufl.edu
}

The Short Baseline Near Detector (SBND) is one of three detectors in the SBN program at Fermilab and will be using LArTPC technology to visualize neutrino interactions. The detector will have an active mass of $\sim 112$ tons of liquid argon and be stationed at $\sim 110 \mathrm{~m}$ away from the Booster Neutrino Beam (BNB) target. The SBND experiment will be playing a crucial role in investigating into the low energy excess observed by the MiniBooNE and LSND experiments, which is the primary objective of the SBN program and will either confirm or rule out the existence of eV-mass scale sterile neutrinos over $5 \sigma$ confidence level. In addition, the experiment will be hosting the world's highest high precision cross section measurements in many different $v_{e}$ and $v_{\mu}$ exclusive channels for $v$-Ar scattering at a few $\mathrm{GeV}$ energy regime. The SBND physics program will also have a special emphasis on some of the BSM physics searches, including milli-charged particles, light dark matter and heavy neutral leptons. The assembly of the different sub systems of the detector such as light detection system, cosmic ray tagger, anode and cathode plane modules, cold electronics and membrane cryostat has already begun in the Fermilab site and many other places and steadily going forward with a targeted timeline of first physics data in early 2023.

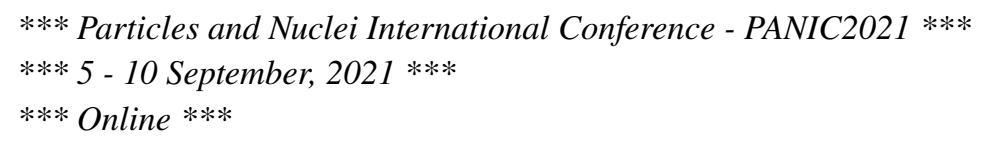

\footnotetext{
${ }^{*}$ Speaker
} 


\section{Short-Baseline Near Detector (SBND)}

The SBND dectector is one of the 3 detectors in the Short-Baseline Neutrino (SBN) program at Fermilab, which is specifically designed to address the puzzle of the existence of eV-mass scale sterile neutrinos, mainly triggered by the observations of MiniBooNE and LSND experiments. The SBND detector is strategically positioned $\sim 110 \mathrm{~m}$ downstream of the Booster Neutrino Beam (BNB) target hall, while the remaining MicroBooNE and ICARUS experiments are $\sim 470 \mathrm{~m}$ and $\sim 600 \mathrm{~m}$ away respectively. All three detectors in the SBN program are using Liquid Argon Time Projection Chamber (LArTPC) techonology to visualize neutrino interactions, where the SBND detector will consist of 2 TPCs having dimensions of $5.0 \mathrm{~m}(\mathrm{~L}) \times 2.0 \mathrm{~m}(\mathrm{~W}) \times 4.0(\mathrm{H})$, leading to an active mass of $\sim 112$ tons.

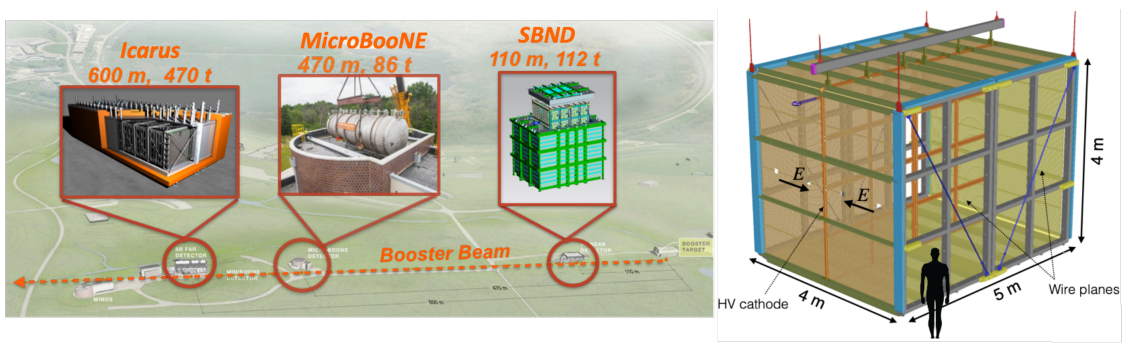

Figure 1: Three detector configuration of the SBN program and a schematic diagram of the SBND detector.

\section{SBND Physics Program}

The SBND experiment will play a crucial role in constraining flux and cross section systematics, which is very important in realizing the main SBN goal of scanning the allowed parameter region, set by previous short-baseline neutrino experiments, over $5 \sigma$ confidence. In addition, the experiment will be collecting $\sim 7$ millions of neutrino interactions in its 3 years of running and performing some of the world's highest statistics cross section measurements in many different $v$-Ar exclusive channels. The other priority of the SBND physics program is to look for some of the BSM processes such as the existence of neutrino tridents, light dark matter, milli-charged particles and heavy neutral leptons.
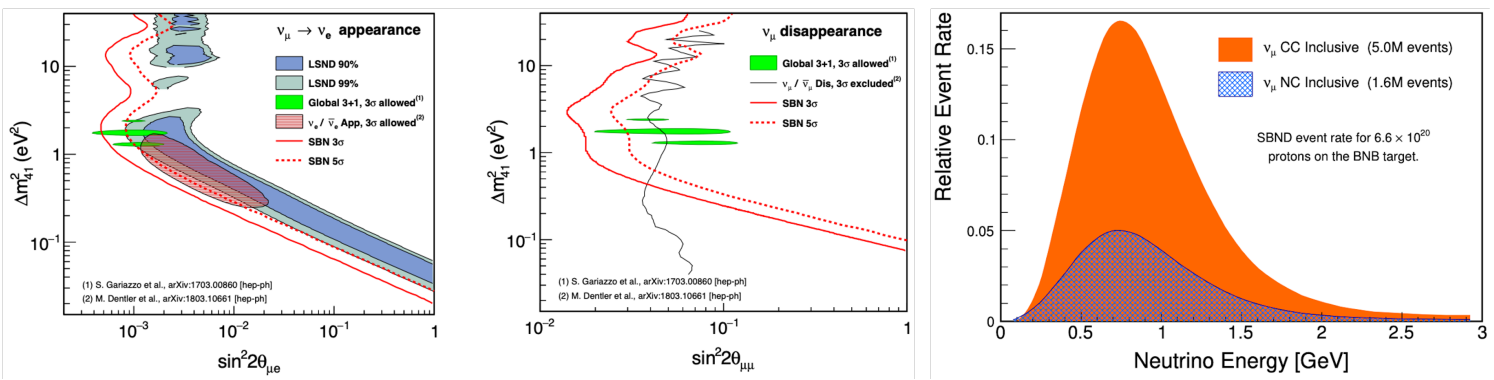

Figure 2: (Left, Middle) $3 \sigma$ and $5 \sigma$ sensitivities of the SBN program in $v_{e}$ appearance and $\mu_{v}$ disappearance channels in 3 year of operations [2]. (Right) Expected charged-current mode and Neutral-current mode $v$ interaction rates in SBND experiment in 3 years of running [2]. 


\section{SBND Light Detection System (LDS)}

The LDS of the SBND experiment consists of 120 Photo Multiplier Tubes (PMT), 192 lighttrapping devices called XARAPUCAs, and light-reflecting Tetraphenyl Butadiene (TPB) coated foils in the cathode. The LDS of SBND serves for many different purposes such as triggering, identifying cosmogenic background, identifying special topologies such as Michel electron decays, and R\&D for upcoming experiments. Currently, all the PMTs are tested, mounted on the PDS boxes, and stored in a light-protected environment until the installation happens. The production of all XArapuca modules is also complete and assembly of the module will soon happen at FNAL, while the TPB coated light-reflecting panels were already installed on the cathode.
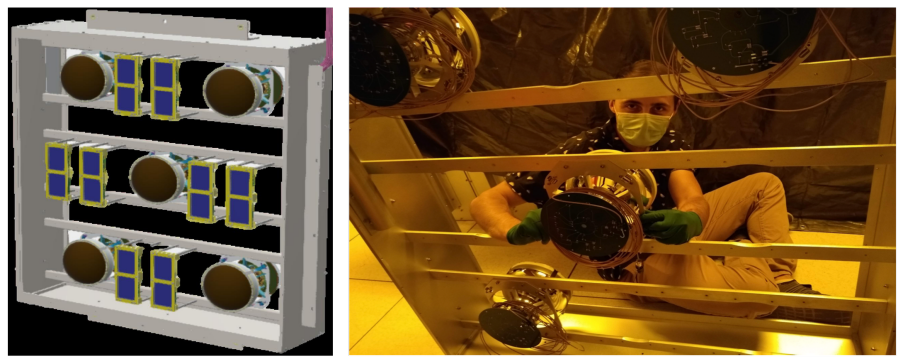

Figure 3: (Left) Schematic diagram of a PDS box in SBND LDS. There are total of 24 of these PDS boxes in SBND LDS. (Right) Mounting PMTs on PDS boxes.

\section{Cosmic Ray Tagger (CRT)}

The SBND CRT consists of a total of 7 taggers, each made up of scintillating strips, arranged in 2 perpendicular planes. The CRT will be providing $\sim 4 \pi$ coverage to the detector in identifying cosmogenic background and is capable of achieving a few nano-seconds level and a few centimeter level timing and coordinate resolutions. Production of all CRT modules are complete and ready to be installed.
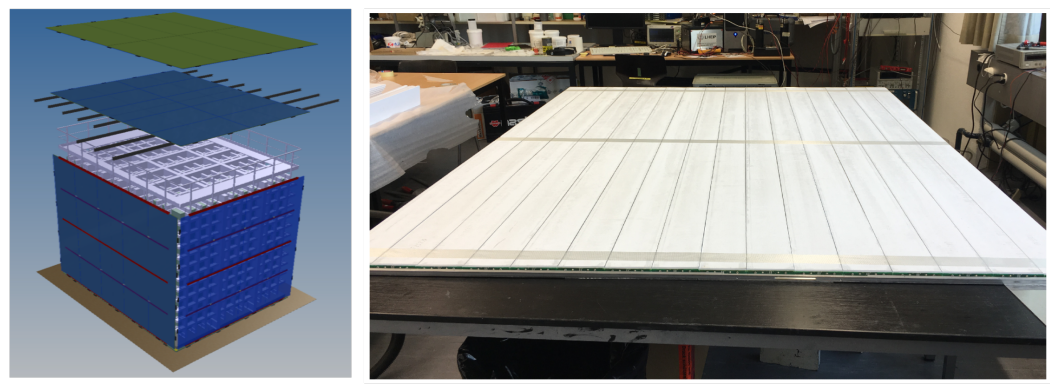

Figure 4: (Left) Schematic diagram of the SBND CRT. (Right) A single CRT plane, made up of scintillating strips.

\section{Anode and Cathode Planes}

The SBND detector will consist of two anode planes, each having 3 wire planes ( 2 induction planes and one collection plane), and a cathode plane. The nominal electric field field between an 
anode plane and the cathode will be $500 \mathrm{~V} / \mathrm{cm}$. At the moment, one anode plane and the cathode plane have already been installed in the detector, where as the assembling of the second anode plane is underway.

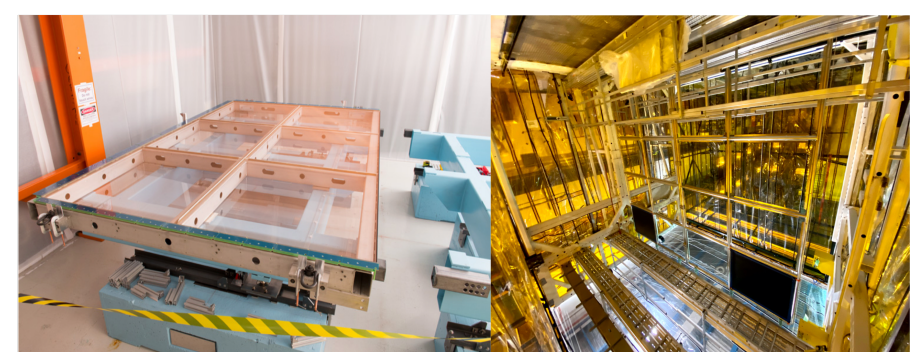

Figure 5: (Left) Completed anode plane before being installed. (Right) Cathode plane after being installed in the detector.

\section{Cold-Electronics}

The SBND will be using special custom-designed, low noise electronics, called cold-electronics (operational at cryogenic temperatures) to further process the analogue signals, collected by anode plane wires. Currently, the production and testing of all cold-electronics components are done and ready to be installed in the detector (Figure 6-Left).

\section{Cryostat}

The SBND will be using membrane-cryostat technology to build its cryostat, which houses the two TPCs. The construction of the warm outer vessel of the cryostat is already complete and inner membrane installation is yet to begin (Figure 6-Right).

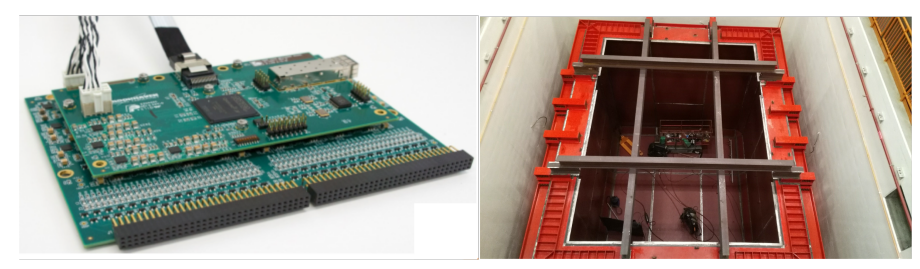

Figure 6: (Left) SBND cold-electronics. (Right) The completed outer warm vessel of the SBND cryostat.

\section{References}

[1] SBND collaboration, "A Proposal for a Three Detector Short-Baseline Neutrino Oscillation Program in the Fermilab Booster Neutrino Beam”, arXiv:1503.01520, 2015.

[2] Pedro A. N. Machado, Ornella Palamara and David W. Schmitz, "The Short-Baseline Neutrino Program at Fermilab", arXiv:1903.04608v1, 2019. 